\title{
Antibody mAb33 from Transduction Laboratories detects human CD95L in ELISA but not in immunoblots
}

\author{
Dear Editor,
}

The expression and function of CD95 ligand (CD95L, FasL, Apo-1L) in normal and pathological immune responses are subject of extensive studies. The analysis of CD95L protein expression relies on antibodies specific for CD95L. Testing CD95L expression by immunoblotting using the monoclonal antibody 33 from Transduction Laboratories (Lexington, KY, USA) raised against CD95L we found CD95L protein in many different cell types even if CD95L transcripts were undetectable by RT-PCR. Because of these contradictory results we further examined mAb33 specificity using a panel of different antibodies including mAb33 for binding to CD95L. Our immunoblotting experiments showed that mAb33 most probably detects a protein expressed by many different cell types but not CD95L. ${ }^{1}$ Referring to the experiments performed by us and by others, ${ }^{1-3}$ Herr et $a l^{4}$ (this issue) reinvestigated the specificity of $\mathrm{mAb3} 3$ using human embryonic kidney carcinoma cell stably transfected with CD95L. Testing the mAb33 specificity by comparison to two other CD95L specific antibodies (NOK-1 ${ }^{5}$ and G247-4) they found that under reducing conditions (immunoblots) mAb33 binds to full-length CD95L. Since these results are different from our data, we repeated our previous experiments using our original and a new batch of mAb33 and several other CD95L specific antibodies as controls. As shown below, these new tests confirmed our earlier experiments with the only exception, that mAb33 was found to detect native CD95L protein in ELISA.

At first we compared by immunoblotting the binding specificities of the old and the new batch of mAb33 to CD95L expressed by transiently transfected 293T cells (Figure 1A). To include also potential effects of tags attached to CD95L two different types of expression vectors were used encoding either human CD95L or a modified form carrying an N-terminal FLAG-tag fused directly in front of the first methionine residue of the cytoplasmic $\mathrm{N}$-terminus. This tagged version of CD95L differs from the recombinant soluble form used by Herr et $a l^{4}$ as the FLAG-tag used here is separated by 100 amino acid residues from the extracellular domain carrying the antigenic epitopes. As expected, under reducing conditions both forms of CD95L were stained in immunoblots with the antibodies G247-4 (Pharmingen, mouse IgG1) and MIKE-2 (Alexis, rat $\lg G$ ), the FLAG specific antibody M2 (Sigma) only stained the tagged form and mock-transfected controls showed no staining (Figure 1A, a). Both batches of mAb33, however, stained a $37 \mathrm{kD}$ signal in untransfected and transfected 293T cells (Figure 1A, b) but not the human endothelial cell lysate that is provided by Transduction Laboratories as a positive control. Although it was used in 2-4-fold excess, this control lysate was also not stained by the CD95L specific antibody G247-4 (Figure 1A, c). Low-level CD95L expression in mock-transfected 293T cells was excluded by CD95L specific RT-PCR using two different primer combinations and total RNA from 293T cells. Samples from human peripheral blood cells or from the NK cell line YT served as controls (Figure $1 \mathrm{~A}, \mathrm{~d})$. Potential differences in the binding affinity between mAb33 and G247-4 were tested by immunoblotting using different amounts $(0.2-20 \mu \mathrm{l})$ of lysates from 293T cells transfected with wildtype CD95L and from controls (Figure 1B). With mAb33, signals were obtained in the 20 and $2 \mu \mathrm{l}$ lysates from transfected and control cells but not with the $0.2 \mu$ lysate. G247-4 detected weak CD95L specific signals in $0.2 \mu \mathrm{l}$ of lysates from transfected cells after 5 min of exposure, which became more intense upon longer exposure (data not shown). Therefore, G247-4 seems to be $10 \times$ more sensitive than mAb33. We conclude from these immunoblot experiments that the binding specificity of mAb33 differs from the specificities of G247-4 and MIKE-2 by at least three aspects. Firstly, mAb33 shows a signal at $37 \mathrm{kD}$ even if CD95L RNA expression is below the detection level of RT-PCR (Figure 1). Secondly, in contrast to G247-4 and MIKE-2, mAb33 staining reveals no differences in CD95L signal intensities when CD95L transfectants are compared to mock-transfected controls although mAb33 seems to be less sensitive than G247-4. Thirdly, G247-4 and MIKE-2 stain multiple CD95L bands that correspond to differentially glycosylated forms of the protein ${ }^{5}$ whereas mAb33 stains only a single band at $37 \mathrm{kD}$. To examine, if any of the signals stained with G247-4, MIKE-2 or other CD95L specific antibodies (like PE62 ${ }^{6}$ ) is identical to the mAb33 specific band, we tested lysates from transfected and from control cells by 2D electrophoresis (Figure 1C). As expected, mAb33 stained an identical signal in lysates from transfected cells and mock-transfected controls (Figure 1C, a, b). When the same blots were incubated with PE62 or G247-4, both antibodies stained identical bands in CD95L transfected cells that were different from the signal revealed with mAb33 (Figure 1C, d, e) and absent in the control lysate PE62 (Figure 1C, f). Since in $2 \mathrm{D}$ electrophoresis $\mathrm{mAb} 33 \mathrm{a}$ reveals a protein with different mobility than the CD95L-specific signal detected by G2474 and PE62, mAb33 has a different binding specificity than G247-4 and PE62. Therefore, mAb33 most probably detects a protein different from CD95L in immunoblots under reducing conditions. This result is also corroborated by the fact that mock-transformed 293T cell controls fail to show CD95L specific signals by RT-PCR. 
A a

G247 MIKE M2

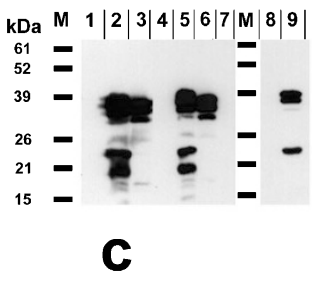

kDa M $1 \mid$\begin{tabular}{l|l|lll|l|l|} 
& 4 & M & 5 & 6 & 7
\end{tabular}
39 -

26 二

21

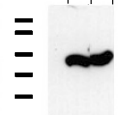

b

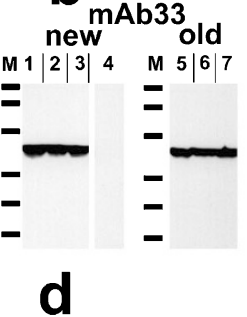

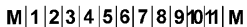

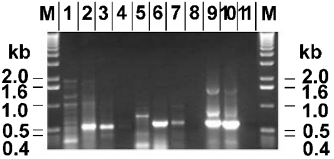

B

mAb33 G247-4 control CD95L CD95L

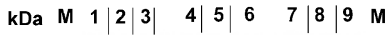

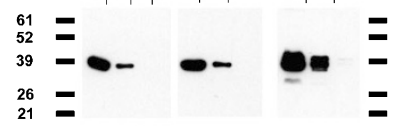

C

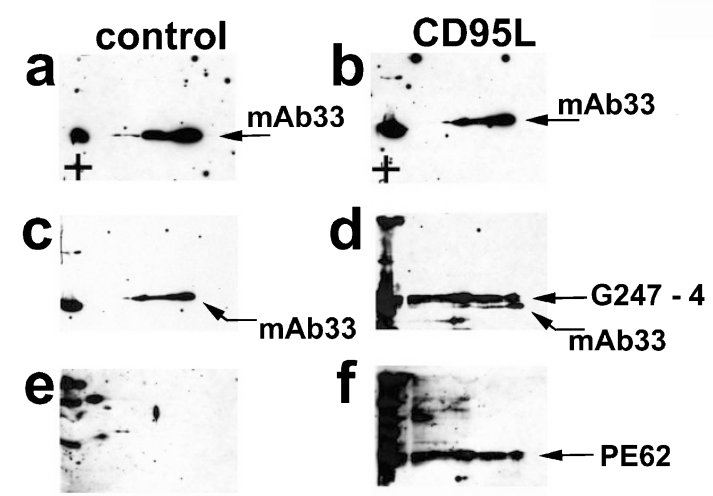

D

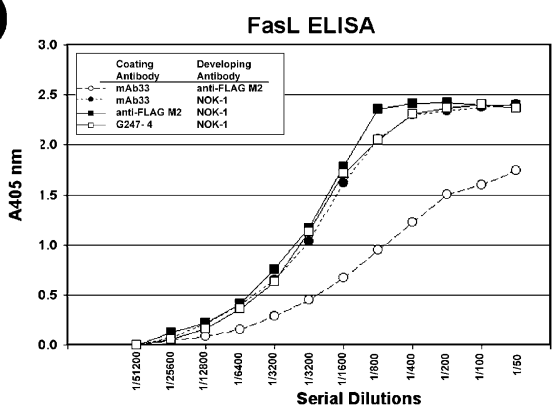

Figure 1 (A) Analysis of CD95L expression by immunoblotting and RT-PCR. (a) 293T cells were transfected with $10 \mu \mathrm{g}$ of wildtype CD95L, CD95L carrying an N-terminal FLAG-tag (FLAG-CD95L) or with vector DNA (mock) by Ca-phosphate precipitation. In a whole cell lysates corresponding to $2-$ $4 \times 10^{6}$ mock- (lanes 1, 4,7), FLAG-CD95L (lanes 2, 5, 9) and wildtype CD95L (lanes 3,6,8) transfected cells were analyzed by SDS-PAGE and immunoblotting using G247-4 (Pharmingen, lanes 1-3; 1:4000 dilution), MIKE-2 (Alexis, lanes 4-7; 1:2000) or the anti-FLAG antibody M2 (Sigma, lanes 8,$9 ; 1: 8000$ ). Blots incubated with G247-4 and M2 were developed with horseradish-peroxidase conjugated goat anti-mouse IgG1 (Southern Biotechn. Ass.) and with horseradish-peroxidase conjugated goat anti-rat IgG (Southern Biotechn. Ass.) for MIKE-2. G247-4 and MIKE-2 show CD95L specific signals only in lysates from cells transfected with the expression vectors but not in the controls. In $\mathrm{b}$, lysates corresponding to $2-4 \times 10^{6}$ mock(lanes 1, 5), FLAG-CD95L (lanes 2,5) and CD95L transfected 293T cells (lanes 3, 7) and from human endothelial cells (provided by Transduction Laboratories as a positive control for CD95L, lane 4) were stained with a new batch (lanes 1-4) and an old batch of mAb33 (Transduction Laboratories). Mab33 detects identical signals in all lysates from 293T cells (lanes 1-3 and 5-7) but no band in the lysate provided by Transduction Labs. In $\mathrm{c}$ and d, mock- and CD95L transfected cells were split into two parts. One part was used for immunoblotting (c, lanes 1, 3, 6: mock-transfected cells, lanes 2, 7: CD95L transfected cells, lanes 4, 5: $20 \mu$ l control lysate from Transduction Laboratories) and stained with G247-4 and the new batch of mAb33. The other part was used for RT-PCR (d) using two different sets of CD95L specific primers. Set A (5'-CGCCGCCGCCACCACTG-3' and 5'GCTGCGGGCCCACATCTGC- $3^{\prime}$ ) was used in lanes $1-4$, set B ( $5^{\prime}$-CCACCGCCACCGCCACCACTA- $3^{\prime}$ and CTGCTGCGGGGCCACATCTGC- $\left.3^{\prime}\right)$ in lanes 5-8). In lanes 9-11 cDNA was amplified with GAPDH specific primers. In lanes 1, 5, 9 PCR was performed with cDNA of 293T cells, in lanes 2, 6, 10 with CDNA from the CD95L expressing NK cell line YT and in lanes 3 and 7 with cDNA from peripheral blood cells. Lanes 4 , 8 , and 11 contained no cDNA. CD95L specific signals were obtained with cDNA from PBL and from YT but not from 293T cells. (B) Binding affinity of G247-4 and mAb33. Twenty $\mu \mathrm{l}$ (lanes 1, 4, 7), $2 \mu \mathrm{l}$ (lanes 2, 5, 8) and $0.2 \mu \mathrm{l}$ (lanes 3, 6, 9) of lysates from mock-transfected (lanes 1-3) and from CD95L transfected 293T cells (lanes 4-9) were analyzed by immunoblotting as described in (A) with mAb33 (lanes 1-6) or with G247-4 (lanes 7-9). After 5 min of exposure, G247-4 (lane 9) but not mAb33 (lane 6) detects a CD95L specific signal in CD95L transfected cells. (C) Analysis of CD95L protein by 2D electrophoresis. Whole cell lysates from $1 \times 10^{6}$ mock transfected and from CD95L transfected 293T cells were separated by NEPHGE in the first dimension and by SDS-PAGE in the 2nd dimension and analyzed separately by immunoblotting in three consecutive steps. Blots were first stained with mAb33 (a, b) and developed with goat anti-mouse IgG1 antibodies coupled to horseradish peroxidase showing identical signals for the mock-control and the CD95L transfected cells. Next, the peroxidase activity was killed with Na-azide and the blots were stained with G247-4 and developed again with goat anti-mouse $\lg G 1$ (c, d). Panels $c$ and $d$ show the reappearing mAb33 signal due to binding of the second antibody but panel $d$ shows a new and different signal detected by G247-4. The peroxidase activity was killed again, the blots were stained in a last round with PE62 ${ }^{6}$ and developed with goat anti-rabbit IgG (e, f). Comparison of blots $d$ and e shows that PE62 and G247-4 detect the same signals in CD95L transfected cells that are not seen the mock-transfected controls and do not match the bands revealed with mAb33. Blocking of G247-4 and PE62-specific epitopes by mAb33 seems to be very unlikely since mAb33 and G247-4 may be used as antibody pairs for CD95L specific ELISA (cf. D). (D) Detection of native CD95L by ELISA. Microtiter wells were coated with mAb33, G247-4 or M2 at $1 \mu \mathrm{g} / \mathrm{ml}$, blocked with PBS $1 \%$ BSA and incubated with serial dilutions of lysates from $4 \times 10^{6}$ mock- and $4 \times 10^{6}$ FLAG-CD95L transfected 293T cells. Binding of CD95L protein was detected either with biotinylated M2 (anti-FLAG, 1:30 000) or with biotinylated NOK-1 (1:50) and streptavidine-phosphatase. Samples were analyzed in an ELISA reader. In the diagram, background staining was subtracted and the OD values were plotted against the serial dilutions of the antigen 
Finally, we compared the binding specificity of mAb33, G247-4 and NOK-1 by ELISA against native, N-terminally FLAG-tagged CD95L present in whole cell lysates of transfected 293T cells. Lysates from mock-transfected cells served as controls. As shown in Figure 1D, all three antibodies bind to native FLAG-tagged CD95L and both combinations (G247-4/NOK-) (mAb33/NOK-1) work equally well as capture and developing antibodies.

In conclusion, the results of our new experiments agree well with our previous data. They show that under reducing conditions (like in immunoblot experiments) mAb33 binds to a protein that differs from CD95L by its electrophoretic mobility in 2D gels and that is present in cells that even do not show CD95L signals by RT-PCR. These results are also in agreement with the results reported by Stokes et $a l^{2}$ but not with the data reported by Herr et $\mathrm{al}^{4}$ (this issue) and by Papoff et $\mathrm{al}^{3}$ and we do not have any explanation for these differences. Analyzing now the binding specificity of mAb33 antibody to native CD95L we came to the same conclusions as presented by Herr et al ${ }^{4}$ showing that mAb33 recognizes non-denatured CD95L in ELISA.

\section{P Fiedler $^{*}{ }^{1}$ and $\mathrm{H}$ Eibel $^{1}$}

${ }^{1}$ Clinical Research Unit for Rheumatology, Division of Rheumatology and Clinical Immunology, Department of Internal Medicine, University Hospital Freiburg, Germany

1. Fiedler $P$ et al. Science (1998) 279: 2015

2. Stokes TA et al. Science (1998) 279: 2015

3. Papoff $G$ et al. Science (1998) 279: 2015

4. Herr I et al. (1999) Cell Death Differ. In press (this issue)

5. Kayagaki $\mathrm{N}$ et al. (1995) J. Exp. Med. 182: 1777

6. Hahne M et al. (1995) Int. Immunol. 7: 1381. 